\title{
Investigation of the Sensitivity to Humidity of an Acrylamide- based Photopolymer Containing N-phenylglycine as a Photoinitiator
}

\author{
Tatsiana Mikulchyk \\ Technological University Dublin, Tatsiana.mikulchyk@tudublin.ie \\ Suzanne Martin \\ Technological University Dublin, suzanne.martin@tudublin.ie \\ Izabela Naydenova \\ Technological University Dublin, izabela.naydenova@tudublin.ie
}

Follow this and additional works at: https://arrow.tudublin.ie/scschphyart

Part of the Optics Commons

\section{Recommended Citation v. 37,pp. 810-815. doi:10.1016/j.optmat.2014.09.012 \\ This Article is brought to you for free and open access by the School of Physics \& Clinical \& Optometric Science at ARROW@TU Dublin. It has been accepted for inclusion in Articles by an authorized administrator of ARROW@TU \\ Dublin. For more information, please contact \\ arrow.admin@tudublin.ie, aisling.coyne@tudublin.ie, gerard.connolly@tudublin.ie. \\ Funder: Irish Research Council Embark Initiative}

Mikulchyk, T., Martin, S., Naydenova, I. (2014). Investigation of the Sensitivity to Humidity of an Acrylamide-based Photopolymer Containing N-phenylglycine as a Photoinitiator. Optical Materials (2014)

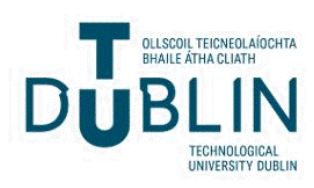




\title{
Investigation of the sensitivity to humidity of an acrylamide-based photopolymer containing N-phenylglycine as a photoinitiator
}

\author{
Tatsiana Mikulchyk, Suzanne Martin and Izabela Naydenova* \\ Centre for Industrial and Engineering Optics, School of Physics, College of Sciences and Health, Dublin Institute of Technology, Kevin Street, \\ Dublin 8, Ireland \\ *izabela.naydenova@dit.ie
}

\begin{abstract}
Sensitivity of holographic recording materials to the relative humidity $(\mathrm{RH})$ of the environment often restricts their use in fabrication of holographic optical elements and other applications. It is important to develop materials with little or no sensitivity to humidity. In this paper the humidity response of transmission gratings recorded in an acrylamide-based photopolymer containing N-phenylglycine (NPG) as a photoinitiator is studied at RH $=20-90 \%$. The hologram is found to be completely insensitive to humidity at RH below $70 \%$ and its diffraction efficiency remains constant. A decrease in diffraction efficiency is observed at $\mathrm{RH}=80-90 \%$ but this decrease is fully reversible, demonstrating quantitatively the NPG photopolymer's excellent resistance to humidity.
\end{abstract}

Keywords: holographic recording materials, acrylamide-based-photopolymer, $\mathrm{N}$-phenylglycine, volume holographic grating, relative humidity.

\section{Introduction}

The application of photopolymers as holographic recording materials is under active study. Photopolymer materials can be used in holographic data storage [1-7], diffractive and refractive optical elements [8,9], solar concentrators [10,11], holographic interferometry [12-16] and holographic sensors [17-19]. Acrylamide-based photopolymers are widely covered in the literature. They contain monomers such as acrylamide (AA) and N, N'-methylene bisacrylamide (BA), electron donor (photoinitiator), a light absorbing dye and a polymer binder such as polyvinyl alcohol (PVA). Triethanolamine (TEA) is usually used as a photoinitiator [20-23]. Photopolymers have a number of advantages: a wide dynamic range, high sensitivity, low scattering, self-processing nature and relatively low cost. However, acrylamide-based photopolymers are known to be very sensitive to humidity due to the highly hygroscopic nature of the components, including the polyacrylamide produced during recording and the PVA binder [24].

In analysing this sensitivity to humidity, it is important to distinguish where possible between reversible change, irreversible change and damage. Stable holograms that exhibit repeatable, reversible change such as a colour change or changes in diffraction efficiency can make useful sensors [17-19]. Holograms that undergo irreversible change that is repeatable and easy to recognise visually can function as indicators, demonstrating visibly that threshold humidity has been reached. However, deterioration of diffraction efficiency and changes in fringe spacing are unacceptable in many applications and both surface damage and delamination are never desirable.

Attempts have been made to improve the environmental stability of acrylamide-based photopolymer layers, using an aerosol sealant as a protective layer [25]. It was found that the sealant increased the humidity stability of the material. However, this sealant created a layer with increased scattering and produced some deterioration in the optical quality of the resulting holographic optical elements. The use of high optical quality glass cover slide is another sealing approach which can be used to decrease the humidity sensitivity of holographic devices. But this is not convenient for every application and adds cost and complexity to the production process.

An investigation of the effect of humidity on the properties of volume reflection and transmission holograms recorded in an acrylamide-based photopolymer layer has been published previously [17-19, 26]. The humidity response of the reflection hologram was characterized at $\mathrm{RH}=5-80 \%$ and in temperature $(\mathrm{T})$ range of $15-50{ }^{\circ} \mathrm{C}$ [17-19]. The material was used to develop a visual indicator of environmental humidity that changed its colour when exposed to different levels of humidity. The colour change occurs due to the changed fringe spacing as the medium gains or losses moisture. The change in the fringe spacing was fully reversible in the relative humidity range under study. In [26] both humidity and temperature effects on properties of transmission gratings recorded in acrylamidebased photopolymer layers were studied. It was found that after exposure to high humidity $(\mathrm{RH}=90 \%)$ diffraction efficiency and Bragg angle changes were irreversible at $\mathrm{T}>16{ }^{\circ} \mathrm{C}$ and reversible below this temperature. These irreversible changes were explained by the irreversible dimensional change in layers and the refractive index modulation. The magnitude of the irreversible change is highly dependent on humidity level, on temperature during 
the humidity exposure and on the time of exposure to humidity. Also, it has been established that photoinitiator such as TEA can affect the stability of the recorded photonic structure at temperature around or above the freezing/melting temperature of TEA. This work showed that the humidity sensitivity of gratings recorded in acrylamide-based photopolymer layers can be exploited for the development of reversible/irreversible humidity indicators, but can also limit the use of these materials in applications where an environmentally stable hologram is needed.

When NPG was used to replace TEA as a photoinitiator previously [27,28], good diffraction efficiencies were achieved and improved surface quality was reported under humid conditions. It was shown that the NPG photopolymer layer appeared unaffected by exposure to $80 \% \mathrm{RH}$ at $\mathrm{T}=25{ }^{\circ} \mathrm{C}$, while the TEA photopolymer layer was visibly damaged. However, no quantitative investigation of the effect of humidity on the properties of holographic optical elements recorded in the NPG material was carried out at that time.

In this paper we investigate the effect of humidity on the diffraction efficiency of transmission gratings recorded in an acrylamide-based photopolymer containing NPG as a photoinitiator (NPG-sample). Experimental data of NPG-samples are compared with the experimental data of acrylamide-based photopolymer containing a commonly used photoinitiator of TEA (TEA-sample) so as to quantitatively compare the humidity sensitivity of the two materials. The humidity response of transmission gratings recorded in photopolymer containing both initiators is also discussed.

\section{Experimental}

Photopolymer solutions with either NPG or TEA as a photoinitiator were prepared using the formulations developed in [27] and [21], correspondingly. The compositions of these solutions are presented in Table 1. The amount of monomers was adjusted in order to have the same monomer concentration in both types of dry layers. The reason for aiming at the same monomer concentration in both compositions is to assure that at the end of holographic recording the amount of polyacrylamide is the same in both types of the layers. Thus, any variation in their response to humidity cannot be directly attributed to the difference in polyacrylamide content. Concentration of the photopolymer components in both types of dry layers is shown in Fig. 1. Methylene blue (MB) was used as a dye in both stock photopolymer solutions. The layers were prepared by casting the photopolymer solution over the levelled glass slide $\left(25 \times 75 \mathrm{~mm}^{2}\right)$ and dried for 24 hours in a dark room at ambient conditions $\left(\mathrm{T}=21 \pm 2{ }^{0} \mathrm{C}\right.$ and $\mathrm{RH}=30$ $40 \%$ ). The amount of deposited photopolymer solutions containing NPG and TEA was $0.8 \mathrm{ml}$ and $0.3 \mathrm{ml}$, respectively. The thickness of the layer was in the range of $50-60 \mu \mathrm{m}$.

Table 1. Photopolymer compositions

\begin{tabular}{lll}
\hline Chemical reagent & NPG-sample & TEA-sample \\
\hline PVA 20\% w/v $(\mathrm{ml})$ & 8 & 8.75 \\
AA $(\mathrm{g})$ & 0.47 & 1.056 \\
BA $(\mathrm{g})$ & 0.093 & 0.192 \\
MB $0.11 \% \mathrm{w} / \mathrm{v}(\mathrm{ml})$ & 1 & 4 \\
TEA $(\mathrm{ml})$ & --- & 1.62 \\
NPG $(\mathrm{g})$ & 0.0267 & --- \\
\hline
\end{tabular}

a)

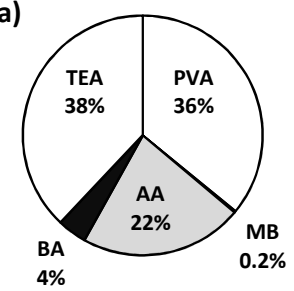

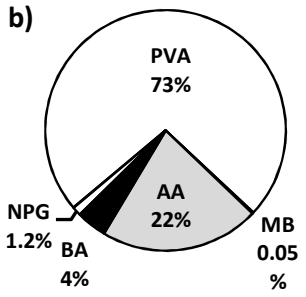

Fig. 1. Photopolymer component concentrations in a) - TEA dry layer and b) - NPG dry layer.

The recordings of transmission volume phase gratings were carried out by using a standard two-beam setup (Fig. 2). A He-Ne laser emitting at a wavelength of $633 \mathrm{~nm}$ was used as a light source. Transmission gratings with 
spatial frequency of approximately 1000 lines/mm and diffraction efficiency of 35 - $40 \%$ were recorded. The total recording intensity was $5 \mathrm{~mW} / \mathrm{cm}^{2}$ and the recording time was $10 \mathrm{sec}$.

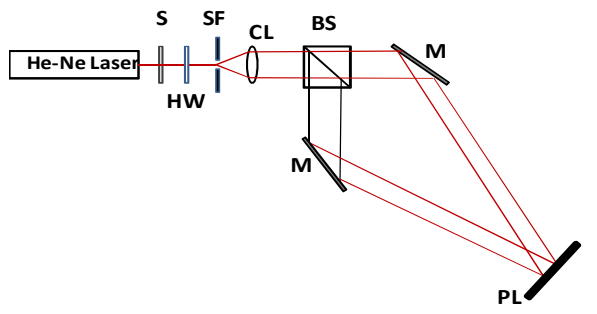

Fig. 2. Recording set-up: S -electronic shutter; HW - half-wave plate; SF - spatial filter; CL collimator; BS - beam splitter; M - mirror; PL - photopolymer layer.

Each grating was characterised by measuring its diffraction efficiency at different incident angles and its Bragg selectivity curve was recorded by a computer controlled system (Fig. 3). A $532 \mathrm{~nm}$ beam from Nd:YVO $\mathrm{Yaser}_{4}$ was employed as a probe beam during the Bragg curve measurements. In order to record the Bragg curve, the grating was placed on a rotation stage which was computer controlled via a motion controller (model Newport ESP300 with angular resolution of $0.001^{\circ}$ ). The intensity of the first-order diffracted beam was monitored by an optical power meter (Newport Model 840). LabVIEW software was used to plot the data of diffraction efficiency in real time as the incident angle of the probe beam was varied $\pm 2^{\circ}$ from the Bragg angle. The diffraction efficiency was defined as the ratio of the diffracted beam intensity and the incident beam intensity.

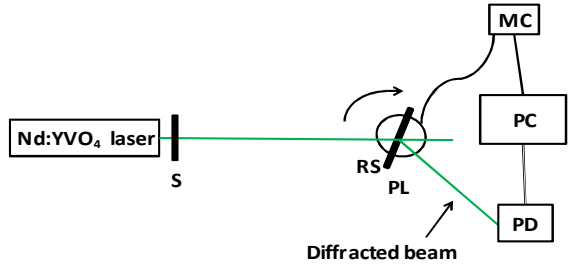

Fig. 3. Set-up for the Bragg selectivity curve recording: S - electronic shutter; RS - rotation stage; PL - photopolymer layer; MC - motion controller; PD - photodetector; PC - computer.

In order to investigate the effect of UV-postexposure on diffraction efficiency of samples and its humidity sensitivity, the samples were bleached under UV light for different amounts of time using a UV Exposure unit (Mega Electronics model LV 202-E).

Different environmental conditions were obtained by a controlled environment chamber with humidity and temperature control system (Electro-tech system, model 5503-11). The chamber is equipped with ETS Series 5000 Microprocessor Controller which is utilized to precisely control the temperature and humidity, to provide accurate measurement and to monitor the chamber environment. The humidity level in the chamber can be reduced down to $10 \%$ RH by the desiccant/pump dehumidification system. An ultrasonic humidification system allows the relative humidity to be increased up to $100 \%$. The heating system can increase the temperature from ambient temperature to $55{ }^{\circ} \mathrm{C}$. The chamber is able to maintain the $\mathrm{RH}$ and temperature with accuracy of $\pm 2 \%$ and $\pm 1{ }^{0} \mathrm{C}$, respectively. After exposure to high humidity the samples were dried for 24 hours at ambient conditions $\left(\mathrm{T}=18 \pm 2{ }^{\circ} \mathrm{C}\right.$ and $\mathrm{RH}=$ $30-40 \%$ ). Then Bragg selectivity curve measurements were carried out again.

\section{Results and Discussion}




\subsection{Dependence of diffraction efficiency on relative humidity levels}

The effect of humidity on the properties of gratings was investigated by exposing the gratings to a range of humidity levels at constant temperature $\left(T=21 \pm 1{ }^{0} \mathrm{C}\right)$. This temperature had been chosen because a significant irreversible decrease of diffraction efficiency has previously been observed at $\mathrm{T}=21 \pm 1{ }^{0} \mathrm{C}$ and $\mathrm{RH}=90 \%$ when working with standard TEA-samples [26].

In order to characterize and compare the diffraction efficiency changes for different samples under humidity exposure, normalized diffraction efficiency was calculated. For every level of RH at which a diffraction efficiency measurement was carried out, the sample was remained at that level for $30 \mathrm{~min}$ in order to allow it to equilibrate with the environment. Both the first-order diffracted beam intensity and the transmitted beam intensity were monitored. In this experiment the diffraction efficiency was calculated as the ratio of the first-order diffracted beam intensity to the sum of the first-order diffracted beam and transmitted beam intensities in order to avoid any scattering losses due to condensation of moisture on the photopolymer surface. In this experiment the normalized diffraction efficiency was defined as the ratio of diffraction efficiency at given humidity level to the diffraction efficiency measured at the start of the experiment at $\mathrm{RH}=20 \%$.

Fig. 4 shows the normalized diffraction efficiency of TEA-sample and NPG-sample versus relative humidity. As can be seen from Fig. 4, in the range of $\mathrm{RH}=20 \%-70 \%$ the normalized diffraction efficiency of NPG-sample is constant, whereas the normalized diffraction efficiency of TEA-sample slightly increases. As found in [26], this increase can be explained by swelling of the TEA-layer in direction perpendicular to the glass slide due to water absorption. It results in the increase of thickness and, hence, the normalized diffraction efficiency increases. Since the normalized diffraction efficiency of NPG-sample remains constant, we can conclude that the NPG-layer absorption of moisture from the environment is either very limited and/or it doesn't cause a detectable swelling, thus, these layers are non-sensitive to humidity changes in this relative humidity range.

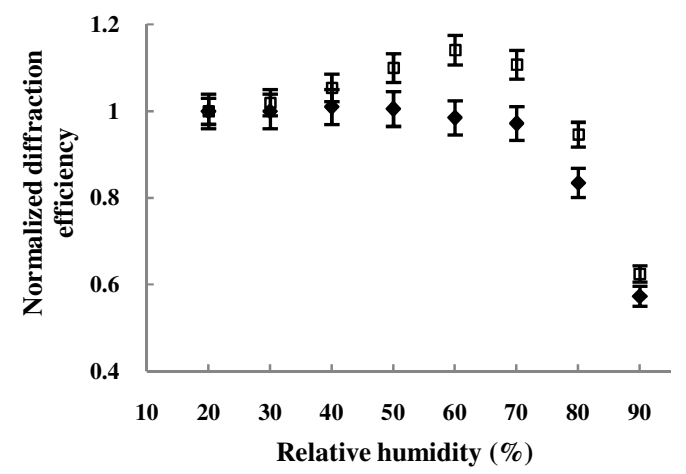

Fig. 4. Normalized diffraction efficiency v/s relative humidity: ( $\bullet$ - NPG-sample and ( $\square)$ - TEA-sample.

Different behaviour of normalized diffraction efficiency of TEA-sample and NPG-sample at $20 \% \leq \mathrm{RH} \leq 70 \%$ can be explained in terms of different ability of these materials to absorb moisture from the environment because of their different compositions. The density of the layers was calculated using the mass of the deposited amount of solution and the thickness of the layer. It has been found that the density of TEA-sample and NPG-sample is approximately $1.03 \mathrm{~g} / \mathrm{cm}^{3}$ and $2.61 \mathrm{~g} / \mathrm{cm}^{3}$, respectively. Thus, the photopolymer layer containing NPG is the denser and less porous material than the photopolymer layer containing TEA. The permeability of NPG-sample is also lower than that of TEA-sample.

At $\mathrm{RH}>70 \%$ the normalized diffraction efficiency of NPG-sample and TEA-sample drops. As shown in [26], the decrease of normalized diffraction efficiency of TEA-sample is irreversible. The change in normalized diffraction efficiency of NPG-sample at high humidity, however, was found to be completely reversible and was studied further in greater detail.

\subsection{Diffraction efficiency dependence on the duration of humidity exposure to $R H=90 \%$}

To investigate the effect of humidity exposure time, changes in normalized diffraction efficiency caused by exposure to $\mathrm{RH}=90 \%$ at $\mathrm{T}=21 \pm 1{ }^{0} \mathrm{C}$ for a set of exposure times have been analysed. In this experiment the diffraction efficiency was initially measured at $\mathrm{RH}=30 \%$, then the sample were exposed to $\mathrm{RH}=90 \%$ for 
different amounts of time. After exposure to high humidity the samples were left for 24 hours at low humidity and the diffraction efficiency was measured again at $\mathrm{RH}=30 \%$. The normalized diffraction efficiency was calculated as the ratio of the diffraction efficiency after and before exposure to high humidity. Fig. 5 shows the normalized diffraction efficiency of NPG-sample and TEA-sample for different humidity exposure times.

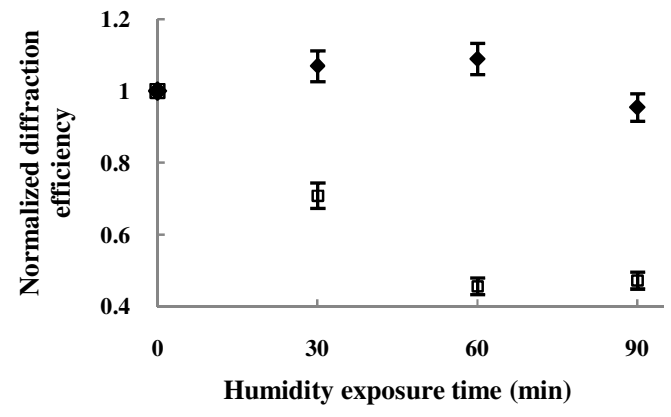

Fig. 5. Normalized diffraction efficiency v/s humidity exposure time: $(\diamond)$ - NPG-sample and ( $\square)$ TEA-sample.

It can be observed from Fig. 5, that normalized diffraction efficiency of NPG-sample does not decrease after exposure to $\mathrm{RH}=90 \%$ for $30 \mathrm{~min}$. Therefore, one can conclude that the decrease of normalized diffraction efficiency of NPG-sample observed during the humidity exposure at RH $=80-90 \%$ for 30 min (Fig. 4) is reversible. The original diffraction efficiency of NPG-sample is fully recovered after exposure to $\mathrm{RH}=90 \%$ for 30 min. It can also be observed that the diffraction efficiency of NPG-sample does not decrease after 60 minutes exposure to $\mathrm{RH}=90 \%$. For longer humidity exposure times the fall off in diffraction efficiency is much smaller for NPG-samples than for TEA-samples, confirming a significant resistance to grating deterioration in very high relative humidity.

\subsection{Effect of exposure to UV-light on the diffraction efficiency}

To better understand the processes behind the observed different dynamics of diffraction efficiency of NPG-sample and TEA-sample under exposure to humidity, the effect of UV-postexposure on TEA-sample and NPG-sample was analysed. For this purpose, after the recordings TEA-samples and NPG-samples were exposed to UV-light for a set period of time. This is normally done in order to remove any residual sensitivity of the layers to light. In TEAsamples TEA plays the role of both photoinitiator and plasticiser. The light absorbing dye is highly mobile over the short fringe distances (in order of $1 \mu \mathrm{m}$ ) and bleaching with UV has a uniform effect on bright and dark fringes. However, some increase of diffraction efficiency is observed for partially polymerized TEA-layers (grating recorded for short exposure times).

The dependence of normalized diffraction efficiency on UV-postexposure time is presented in Fig. 6 for both formulations. In this experiment the normalized diffraction efficiency has been defined as the ratio of diffraction efficiency measured after certain UV-postexposure time to the diffraction efficiency measured before UVpostexposure.

As can be seen from Fig. 6, UV-postexposure for 30 min causes increase of normalized diffraction efficiency of TEA-sample by $60 \%$, whereas the normalized diffraction efficiency of NPG-sample is decreased by $60 \%$. The normalized diffraction efficiency of TEA-sample reaches saturation after $15 \mathrm{~min}$ UV-postexposure, while the normalized diffraction efficiency of NPG-sample still decreases after $15 \mathrm{~min}$. 


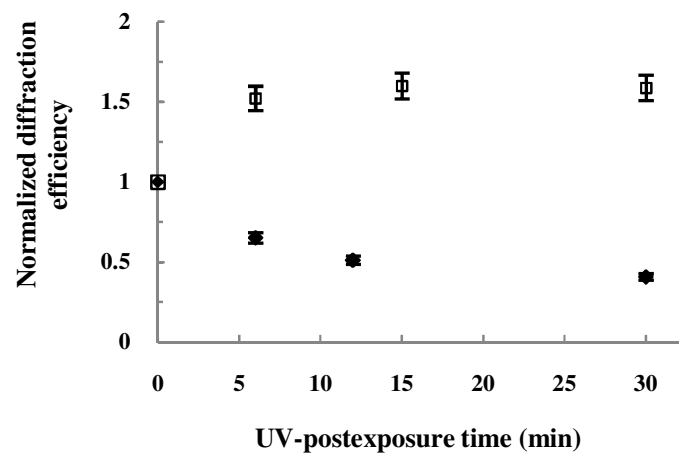

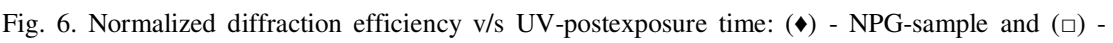
TEA-sample.

The different dynamics of normalized diffraction efficiency of NPG-sample and TEA-sample under UVpostexposure can be explained in terms of monomer diffusion rates inside these materials. It has been observed previously that TEA affects the diffusion rates of the photopolymer components [29]. Since the TEA plays the role of a plastisizer, it increases the diffusion rates. During holographic recording the monomers diffuse from dark to bright fringes where they are polymerized. Under UV-light exposure, which is uniform and has no fringe pattern, all remaining monomers are polymerized, including those remaining in the dark fringe regions. The increase of the diffraction efficiency in the case of TEA-sample implies that the refractive index modulation increases under UVpostexposure. However, UV-light exposure causes an overall deacrease in the diffraction efficiency of NPG-sample. This could be attributed to the very slow monomer diffusion rate in the NPG-sample The restricted diffusion during the recording process may lead to the gratings having the opposite refrective index distribution to the one in the TEA-sample, causing UV polymerization to have the opposite effect on the diffraction efficiency. This is further discussed in section 3.5 where two scenarios assuming fast and slow monomer diffusion are considered for the explanation of both UV-postexposure results and the influence of high humidity on the diffraction efficiency of TEA-sample and NPG-sample, respectively.

\subsection{The humidity response/sensitivity of $U V$-stabilised samples}

Humidity sensitivity of NPG-samples after UV-postexposure for $30 \mathrm{~min}$ has been further investigated. Changes in normalized diffraction efficiency caused by exposure to $\mathrm{RH}=90 \%$ at $\mathrm{T}=21 \pm 1{ }^{0} \mathrm{C}$ for a set period of time have been analysed. In this experiment the normalized diffraction efficiency was defined as in section 3.2. Fig. 7 shows the normalized diffraction efficiency of samples with UV-postexposure for different humidity exposure times.

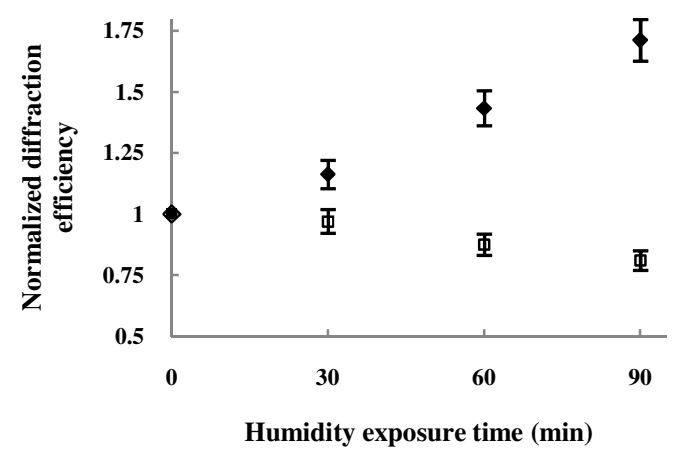

Fig. 7. Normalized diffraction efficiency of samples with UV-postexposure v/s humidity exposure time: $(\diamond)$ - NPG-sample and ( $\square)$ - TEA-sample.

As shown in Fig. 7, the effect of duration of humidity exposure on normalized diffraction efficiency of NPGsamples and TEA-samples is significantly different. There is a $20 \%$ decrease of normalized diffraction efficiency of TEA-sample due to exposure to $\mathrm{RH}=90 \%$ for $90 \mathrm{~min}$. In the case of NPG-sample the normalized diffraction 
efficiency significantly increases with the exposure time. Thus, exposure to $\mathrm{RH}=90 \%$ leads to close to linear increase of the normalized diffraction efficiency reaching $70 \%$ for $90 \mathrm{~min}$ exposure. To check if the increase is irreversible the diffraction efficiency measurements have been carried out again 4 days after exposure to high humidity and it was observed that this increase of diffraction efficiency was permanent. In order to understand this it is important to note that the diffraction efficiencies are normalised to the diffraction efficency value at the start of humidity exposure. For bleached NPG-samples this has been reduced by the bleaching process and what is being obseved is a recovery of some of that reduction.

\subsection{Mechanism of obtaining refractive index modulation in photopolymers}

According to the coupled wave theory [30], the diffraction efficiency of the holographic grating depends on the refractive index modulation of the medium where this holographic grating was recorded. The formation of the refractive index change in the photopolymer containing TEA as a photoinitiator has been investigated previously [31]. Based on a model published in [32] it has been proposed that changes in density and molar refraction are the main contributors to the refractive index change created during holographic recording. Exposure to light induces the polymerization process in the bright regions, and the polymer regions with higher density are formed. The dark regions contain monomers and have lower density. Thus, we have a periodic structure of bright and dark regions with different density. At the same time, these regions have different molar refraction. On polymerization in each monomer unit a carbon-carbon double bond having higher molar refraction is replaced by two carbon-carbon single bonds having lower molar refraction. The periodic conversion of double to single bonds also causes refractive index modulation which is shifted with respect to the modulation due to density variation by 180 degrees (Fig.8).

Fig. 8 shows the two processes contributing to the final refractive index modulation - change in molecular refraction and change in density. Fig. 8a presents the two processes and the resulting refractive index modulation in the TEA-sample. It is assumed that the recording is carried out in a regime when most of the monomer molecules manage to move from dark to bright fringe regions (fast diffusion regime). In the fast diffusion regime, mass transport of material from dark to bright fringe areas is the main contributor to the refractive index change [31]. During the holographic recording, a periodic structure of bright and dark regions with different density and molar refraction is created. The periodic variation of density is shifted by 180 degrees in respect of the periodic variation of molar refraction. The density changes outweigh the changes in molar refraction and the bright fringe areas have higher refractive index. This is proved by the experimental data on the effect of UV-light on the diffraction efficiency (Fig. 6). As can be seen from Fig. 6, exposure to UV light after holographic recording causes increase of the diffraction efficiency of TEA-sample. The diffraction efficiency increase can be explained by overall increase of the refractive index modulation due to "erasing" bond conversion variation.

Fig. $8 \mathrm{~b}$ shows the mechanism of obtaining refractive index modulation in NPG-sample. It is assumed that the monomer molecules diffuse slowly and only a small fraction of them reach the bright fringe area (slow diffusion regime). Based on our experimental results discussed in section 3.2-3.4, we can suppose that in the case of NPGsample (slow diffusion regime), structural changes in the monomer molecule play a major role in the formation of refractive index modulation. Changes in molecular refraction outweigh the density changes and, thus, bright fringe areas have lower refractive index in NPG-sample. Exposure to UV light after holographic recording causes changes in the refractive index modulation due to bond conversion and, thus, leads to the decrease of the refractive index modulation in NPG-sample. Exposure to high humidity which allows the polymer chains to move from bright to dark fringe areas can then lead to a decrease in the refractive index in the bright fringe areas and, hence, decrease in diffraction efficiency of TEA-sample. In case of NPG-sample, exposure to high humidity leads to increase in refractive index in dark fringe areas and increase in diffraction efficiency (as observed in Fig. 7). 

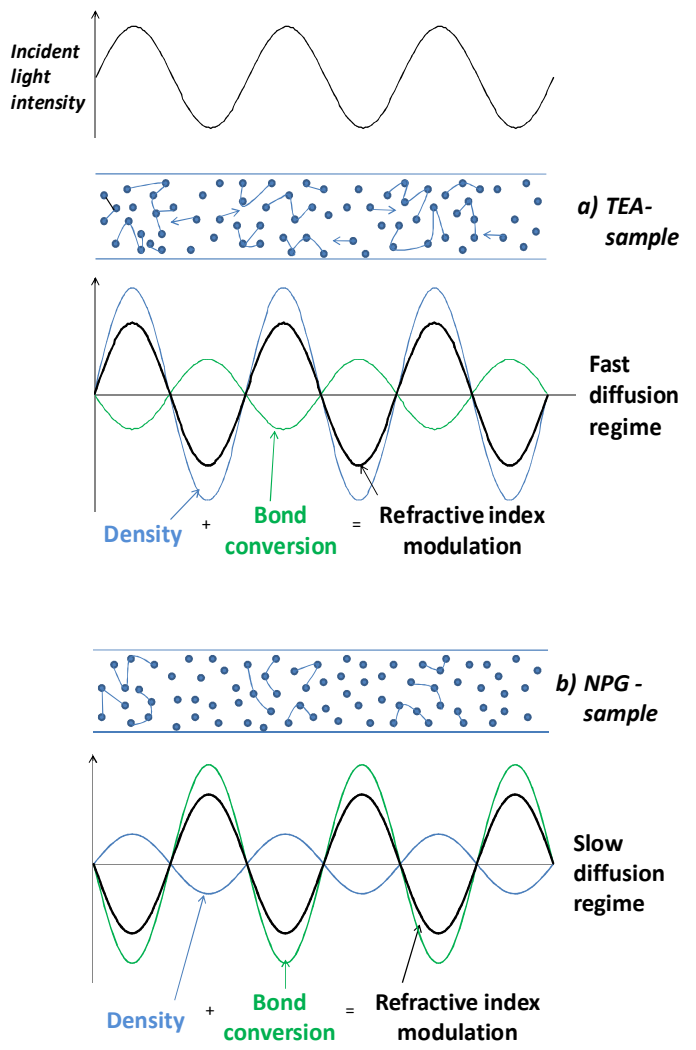

Fig. 8. Mechanism of obtaining refractive index modulation in a) TEA-sample and b) NPG-sample.

\subsection{Influence of triethanolamine on humidity sensitivity of photopolymer layers}

To investigate the effect of TEA on humidity sensitivity of the layers, normalized diffraction efficiency of NPGsamples and TEA-samples with different concentration of TEA was analyzed. In this experiment, two additional types of samples were prepared. The first type was NPG-sample in which the concentration of TEA in the dry layer was $9 \%$. It was made by adding one-tenth of proposed in [21] amount of TEA to the NPG-sample stock solution. The second type of the layer was the TEA-sample containing the half of the amount of TEA proposed in [21]. So, the concentration of TEA in this TEA-sample was $23 \%$. After the recording, all samples were exposed to UV-light for $30 \mathrm{~min}$ and placed into the humidity chamber at $\mathrm{RH}=90 \%$ for $60 \mathrm{~min}$. Normalized diffraction efficiency calculated as in section 3.2 versus concentration of TEA is shown in Fig. 9.

As seen from Fig. 9, the normalized diffraction efficiency of NPG-sample containing 9\% of TEA is significantly less than the normalized diffraction efficiency of the pure NPG-sample. The increase in normalized diffraction efficiency caused by humidity exposure is only few percent, while in the pure NPG-sample the increase is above $40 \%$. The normalized diffraction efficiency of TEA-samples with $23 \%$ and $38 \%$ of TEA is very similar. These results reveal that changes in diffraction efficiency caused by exposure to high humidity depend on the concentration of TEA in photopolymer layers. The higher the concentration of TEA, the lower the normalized diffraction efficiency and the higher the humidity sensitivity of photopolymer layers. Thereby, it is possible to alter the humidity sensitivity of the photopolymer varying the concentration of TEA. 


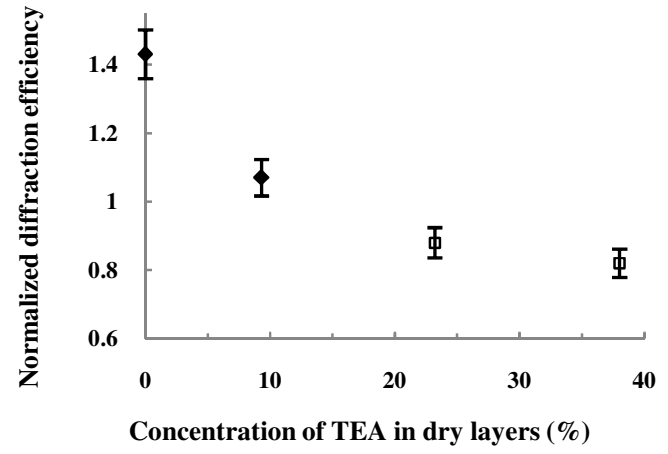

Fig. 9. Normalized diffraction efficiency v/s concentration of TEA in the dry layers: $(\bullet)$ - NPGsamples and ( $\square)$ - TEA-samples.

\section{Conclusions}

The investigation of humidity sensitivity of acrylamide-based photopolymer containing NPG as a photoinitiator has been carried out in a wide range of relative humidity $\mathrm{RH}=20-90 \%$. In the relative humidity range $\mathrm{RH}=20-70$ $\%$ NPG-sample is non-sensitive to humidity change and the normalized diffraction efficiency of NPG-samples is found to be constant. The normalized diffraction efficiency of NPG-samples is recovered after exposure to RH $=90$ $\%$ for $30 \mathrm{~min}$. Thus, it has established that decrease in normalized diffraction efficiency observed at $\mathrm{RH}=80-90 \%$ is fully reversible. The observed low humidity sensitivity of acrylamide-based photopolymer containing NPG is encouraging for applications where non-sensitive to humidity material is needed.

Based on the influence of UV-postexposure and exposure to $\mathrm{RH}=90 \%$ on diffraction efficiency of gratings recorded in TEA-samples and NPG-samples the following model for the refractive index modulation can be proposed. The TEA-sample records in regime of fast monomer diffusion, the main contributor to the refractive index modulation is the density change, and the refractive index modulation has its maximum in the bright fringe regions. The NPG-sample records in regime of slow monomer diffusion, the main contributor to the refractive index modulation is the change in molar refraction, and the refractive index modulation has its maximum in the dark regions.

Also, it has been shown that it is possible to alter the humidity sensitivity of the photopolymer by variation of the concentration of TEA.

\section{Acknowledgements}

The authors would like to acknowledge the financial support provided by the Irish Research Council Embark Initiative and Ulysses Programme, the COST 1205 Action, and also thank the FOCAS Research Institute for providing research facilities.

\section{References and links}

[1] J. Ashley, M. Bernal, G. Burr, H. Coufal, H. Guenther, J. Hoffnagle, C. Jefferson, B. Marcus, R. MacFarlane, R. Shelby, G. Sincerbox, Holographic data storage technology, IBM J. Res. \& Dev. 44(3) (2000) 341-368.

[2] H. Yao, M. Huang, Z. Chen, L. Hou, F. Gan, Optimization of two-monomer-based photopolymer used for holographic recording, Mater. Lett. 56(1-2) (2002) 3-8.

[3] E. Fernandez, C. Garcia, I. Pascual, M. Ortuno, S. Gallego, A. Belendez, Optimization of a thick polyvinyl alcohol-acrylamide photopolymer for data storage using a combination of angular and peristrophic holographic multiplexing, Appl. Optics 45(29) (2006) 7661-7666.

[4] S. Gallego, A. Marquez, S. Marini, E. Fernandez, M. Ortuno, I. Pascual, In dark analysis of PVA/AA materials at very low spatial frequencies: phase modulation evolution and diffusion estimation, Opt. Express 17(20) (2009) 18279-18291.

[5] H. Sherif, I. Naydenova, S. Martin, C. McGinn, V. Toal, Characterisation of an acrylamide-based photopolymer for data storage utilizing holographic angular multiplexing, J. Opt. A: Pure Appl. Opt. 7 (2005) 255-260.

[6] R. Castagna, F. Vita, D. E. Lucchetta, L. Criante, F. Simoni, Superior-performance polymeric composite materials for high-density optical data storage, Adv. Mater. 21(5) (2009) 589-592.

[7] T. Sabel, S. Orlic, K. Pfeiffer, U. Ostrzinski, G. Grützner, Free-surface photopolymerizable recording material for volume holography, Opt. Mater. Express 3(3) (2013) 329-338.

[8] F. O’Neill, A. Carr, S. Daniels, M. Gleeson, J. Kelly, J. Lawrence, J. Sheridan, Refractive elements produced in photopolymer layers, J. Mater. Sci. 40(15) (2005) 4129-4132. 
[9] A. Marquez, S. Gallego, M. Ortuno, E. Fernandez, M. Alvarez, A. Belendez, I. Pascual, Generation of diffractive optical elements onto a photopolymer using a liquid crystal display, Proc. SPIE 7717(2010) 77170D.

[10] I. Naydenova, H. Akbari, C. Dalton, M. Yahya so M. Ilyas, C. Pang Tee Wei, V. Toal, S. Martin, Photopolymer Holographic Optical Elements for Application in Solar Energy Concentrators, in Holography - Basic Principles and Contemporary Applications, E. Mihaylova (Ed.), InTech, 2013.

[11] H. Akbari, I. Naydenova, S. Martin, Using acrylamide-based photopolymers for fabrication of holographic optical elements in solar energy applications, Appl. Optics 53(7) (2014) 1343-1353.

[12] S. Guntaka, V. Toal, S. Martin, Holographically recorded photopolymer diffractive optical element for holographic and electronic specklepattern interferometry, Appl. Optics 41(35) (2002) 7475-7479.

[13] V. Bavigadda, J. Jallapuram, E. Mihaylova, V. Toal, Electronic speckle pattern interferometer using holographic optical elements for vibration measurements, Opt. Lett. 35(19) (2010) 3273-3275.

[14] S. Gallego, A. Marquez, D. Mendez, M. Ortuno, C. Neipp, E. Fernandez, E. Pascual, A. Belendez, Analysis of PVA/AA based photopolymers at the zero spatial frequency limit using interferometric methods, Appl. Optics 47(14) (2008) $2557-2563$.

[15] X. Guo, J. Zhu, C. Xia, J. Li, and L. Chen, Characterization of a real-time high sensitivity photopolymer for holographic display and holographic interferometry, Proc. SPIE 5636 (2005) 528-537.

[16] J. Zhu, G. Wang, Y. Hao, B. Xie, A. Cheng, Highly sensitive and spatially resolved polyvinyl alcohol/acrylamide photopolymer for real time holographic applications, Opt. Express 18(17) (2010) 18106-18112.

[17] I. Naydenova, R. Jallapuram, V. Toal, S. Martin, A visual indication of environmental humidity using a colour changing hologram recorded in a self-developing photopolymer, Appl. Phys. Lett. 92(3) (2008) 031109.

[18] I. Naydenova, R. Jallapuram, V. Toal, S. Martin, Characterization of the humidity and temperature responses of a reflection hologram recorded in acrylamide-based photopolymer, Sensor Actuat B-Chem. 139(1) (2009) 35-38.

[19] I. Naydenova, R. Jallapuram, S. Martin, V. Toal, Holographic humidity sensors, in: C. Okada (Ed.), Humidity Sensors: Types, Nanomaterials and Environmental Monitoring, Nova Science Publishers, 2011.

[20] Y. Boiko, V. Solovjev, S. Calixto, D-J. Lougnot, Dry photopolymer films for computer-generated infrared radiation focusing elements, Appl. Optics 33(5) (1994) 787-792.

[21] S. Martin, P. Leclere, Y. Renotte, V. Toal, Y. Lion, Characterization of an acrylamide-based dry photopolymer holographic recording material, Opt. Eng. 33(12) (1994) 3942-3946.

[22] S. Blaya, L. Carretero, R. Madrigal, A. Fimia, Optimization of a photopolymerizable holographic recording material based on polyvinylalcohol using angular responses, Opt. Mater. 23(3-4) (2003) 529-538.

[23] L. Hai, L. Ruo-Ping, S. Cai-Xia, X. Yong, T. Dao-Guang, H. Ming-Ju, Holographic property of photopolymers with different amine photoinitiators, Chinese Phys. B 19(2) (2010) 024212.

[24] R. Spooncer, F. Al-Ramadhan, B. Jones, A humidity sensor using a wavelength-dependent holographic filter with fibre optic links, Int. J. Optoelectron. 7(3) (1992) 449-452.

[25] F. Neill, J. Lawrence, J. Sheridan, Improvement of holographic recording material using aerosol sealant, J. Opt. A-Pure Appl. Op. 3(1) (2001) 20-25.

[26] T. Mikulchyk, S. Martin, I. Naydenova, Humidity and temperature effect on properties of transmission gratings recorded in PVA/AA-based photopolymer layers, J. Opt. 15 (2013) 105301.

[27] Q. Gong, S. Wang, M. Huang, F. Gan, A humidity-resistant highly sensitive holographic photopolymerizable dry film, Mater. Lett. 59(23) (2005) 2969-2972.

[28] Q. Gong, S. Wang, M. Huang, Y. Dong, F. Gan, Effects of dyes and initiators on the holographic data storage properties of photopolymer, Proc. SPIE 5966 (2005) 59660P.

[29] C. Feely, The development and characterization of a red-sensitized photopolymer holographic recording material (Doctoral Thesis), Dublin Institute of Technology, 1998.

[30] H. Kogelnic, Coupled wave theory for thick hologram gratings, Bell Syst. Tech. J. 48(9) (1969) 2909-2947.

[31] S. Martin, A new photopolymer recording material for holographic applications: photochemical and holographic studies towards an optimized system (Doctoral Thesis), Dublin Institute of Technology, 1995.

[32] W. Tomlinson, E. Chandross, Organic photochemical refractive index image recording systems, in: J. N. Pitts, G. S. Hammond, K. Gollnick, D. Grosjean (Eds.), Advances in Photochemistry, twelfth ed., John Wiley \& Sons, 1980. 